\title{
Further Results on Capacitated Network Design Games
}

\author{
Thomas Erlebach and Matthew Radoja \\ Department of Computer Science, University of Leicester, England \\ $\{$ te17,mr193\}@leicester.ac.uk
}

\begin{abstract}
In a capacitated network design game, each of $n$ players selects a path from her source to her sink. The cost of each edge is shared equally among the players using the edge. Every edge has a finite capacity that limits the number of players using the edge. We study the price of stability for such games with respect to the max-cost objective, i.e., the maximum cost paid by any player. We show that the price of stability is $O(n)$ for symmetric games, and this bound is tight. Furthermore, we show that the price of stability for asymmetric games can be $\Omega(n \log n)$, matching the previously known upper bound. We also prove that the convergence time of best response dynamics cannot be bounded by any function of $n$.
\end{abstract}

\section{Introduction}

The quantification of the inefficiency of Nash equilibria has received considerable attention in recent years. The concept of the price of anarchy, measuring the inefficiency of the worst Nash equilibrium (NE) of a given game compared to a social optimum, was introduced by Koutsoupias and Papadimitriou [5], who called it the coordination ratio. The price of stability, measuring the inefficiency of the best NE of a given game, was first studied by Schulz et al. [9], under the name optimistic price of anarchy. Games for which these measures have been studied include scheduling games [5], routing games [8], network design games [2], and capacitated network design games [4]. Apart from the study of the inefficiency of $\mathrm{NE}$, one is also interested in the convergence time of best response dynamics (BRD), i.e., the process that starts with an arbitrary strategy profile and iteratively allows one of the players to update her strategy to one that optimises her cost given the current strategies of all the other players.

In a capacitated network design game, we are given an undirected graph with edge costs and edge capacities, and each of the $n$ players selects a path from her source to her destination. The cost of an edge is shared equally among the players using the edge. Each player aims to minimise her own cost. A capacitated network design game is symmetric if all players share the same source and the same destination, and asymmetric otherwise. As the social optimum, one usually considers the best strategy profile with respect to sum-cost (total cost of all players) or max-cost (maximum cost of any player). 
Feldman and Ron [4] studied symmetric capacitated network design games and considered instances where the underlying graph is a set of parallel links, a series-parallel graph, or an arbitrary graph. They gave tight bounds on the maximum price of stability and the maximum price of anarchy for all cases for both the max-cost objective and the sum-cost objective, except for the price of stability with respect to max-cost for arbitrary graphs. For the latter case, they showed an upper bound of $O(n \log n)$ and a lower bound of $\Omega(n)$, and they posed closing this gap as an open problem. They also analysed BRD and showed that there are symmetric capacitated network design games where convergence requires $\Omega\left(n^{3 / 2}\right)$ steps, contrary to the uncapacitated version of symmetric network design games where BRD always converge in at most $n$ steps.

Our contribution. For symmetric games with $n$ players, we show that the price of stability with respect to max-cost is $O(n)$. This bound is tight, as implied by the matching lower bound from [4], and hence resolves the open problem posed by Feldman and Ron. A standard proof technique for bounding the price of stability is to bound the increase in social cost during best response dynamics starting from the optimal strategy profile. We show that this technique does not work in our case, as best response dynamics starting from the optimal strategy profile can actually increase the max-cost by a factor of $\Theta(n \log n)$. Therefore, we use a different approach to bound the price of stability, which may be of independent interest. For asymmetric games with $n$ players, we show that the price of stability can be $\Omega(n \log n)$, matching the previously known upper bound. We also analyse BRD and show that the number of update steps required to converge to a NE cannot be bounded by any function of $n$, even for symmetric games. Our construction does not depend on the order in which players are allowed to update their strategies. Furthermore, we observe that the cost of a player can grow by an arbitrary factor (not bounded by any function of $n$ ) during BRD.

Outline. The remainder of the paper is structured as follows. Section 2 discusses related work. Section 3 gives formal definitions and other preliminaries. Our results on BRD and on the price of stability with respect to max-cost are presented in Sections 4 and 5, respectively. Section 6 suggests possible directions for future research.

\section{Related Work}

We discuss only related work on network design games and refer to [7] for general background on algorithmic game theory and the inefficiency of equilibria for different types of games. Network design games with fair cost sharing, where the cost of an edge is distributed to all players using the edge in equal shares, were first studied by Anshelevich et al. [2]. They observe that these games are potential games [6] and therefore always have a NE in pure strategies, and BRD converge to such a NE. For asymmetric, uncapacitated network design games on directed graphs, they show that the price of stability with respect to sum-cost is at most $\mathrm{H}(n)=\Theta(\log n)$, where $\mathrm{H}(n)=\sum_{i=1}^{n} 1 / i$ denotes the $n$-th harmonic number. 
They prove this result by considering a potential function that decreases with every improving move of a player and using it to show that BRD from an optimal strategy profile must lead to a NE whose sum-cost is at most $\mathrm{H}(n)$ times the sum-cost of the starting profile. We will use the same potential function several times in this paper. They also show that the upper bound of $\mathrm{H}(n)$ on the price of stability for sum-cost holds for several generalisations, including capacitated network design games. Regarding BRD, they construct a network design game with $n$ players where the convergence to a NE may take a number of steps that is exponential in $n$ (if players make their improving moves in a certain order). It is also known that in symmetric uncapacitated network design games, BRD converge to a $\mathrm{NE}$ in at most $n$ steps, as the best response for the first update will also be the best response for all other players [4].

The price of stability of uncapacitated network design games with respect to sum-cost for undirected networks is still open. The best known lower bounds are constant and the best known upper bound is $\left(1-\Theta\left(1 / n^{4}\right)\right) \mathrm{H}(n)$, showing that the maximum price of stability for undirected networks is smaller than it is for directed networks, see [3] and the references given there.

As already noted in [4], it is easy to see that the price of stability is 1 for both sum-cost and max-cost for symmetric network design games without capacities, since the strategy profile where all players choose the same minimum-cost path from the common source to the common destination is a NE and also the social optimum.

Feldman and Ron [4] present a comprehensive study of symmetric capacitated network design games in undirected networks. They show that the price of anarchy is unbounded for both sum-cost and max-cost in general networks, but is bounded by $O(n)$ for parallel links and series-parallel networks. For the price of stability with respect to sum-cost, they show a bound of $O(\log n)$ that is tight even for parallel links. For the price of stability with respect to max-cost, they give tight bounds of $O(n)$ for parallel links and series-parallel networks, but for arbitrary networks their upper bound of $O(n \log n)$ leaves a gap to the lower bound of $\Omega(n)$.

\section{Model and Definitions}

Capacitated Network Design Games. We consider capacitated network design games, also known as capacitated cost sharing (CCS) games and referred to as CCS games in the following. These games are discrete. All players (or agents) have perfect knowledge of their strategy space and the cost, ceterus paribus, associated with each strategy. For some directed or undirected graph $G=(V, E)$, each player in a set of $n$ must establish a connection between their source and sink nodes. Every edge $e \in E$ has cost $p(e) \in \mathbb{R}^{\geq 0}$ and capacity $c(e) \in \mathbb{N}$. We also write $p_{e}$ for $p(e)$ and $c_{e}$ for $c(e)$. Let $[n]$ denote the set $\{1,2, \ldots, n\}$. The game can be represented as the tuple

$$
\Delta=\left\langle n, G=(V, E),\left\{s_{i}\right\}_{i \in[n]},\left\{t_{i}\right\}_{i \in[n]},\left\{p_{e}\right\}_{e \in E},\left\{c_{e}\right\}_{e \in E}\right\rangle .
$$


The set of agent $i$ 's strategies is the set of $s_{i}-t_{i}$ paths in $G$. We usually denote the strategy of agent $i$ by $S_{i}$ and the strategy profile of all $n$ players as $S$. By $S_{-i}$ we denote the joint action of all agents except $i$ in some profile $S$. As we are considering capacitated networks, a feasibility issue arises. A strategy profile $S=\left(S_{1}, \ldots, S_{n}\right)$ is feasible if $x_{e}(S) \leq c_{e}$ for all $e \in E$, where $x_{e}(S)=\mid\{i$ : $\left.e \in S_{i}\right\} \mid$ denotes the number of agents that use $e$ in their path. Throughout this paper we will only consider feasible games, i.e., games for which there is at least one feasible strategy profile. We do not impose any restrictions on the network topology, i.e., we allow arbitrary graphs. If we require that all $n$ players have the same source and the same destination, we call the game symmetric, and asymmetric otherwise.

The price of an edge to an individual player using the edge is an equal slice of its cost which is shared among all the players using the edge. This fair cost division scheme is derived from the Shapley value, and is one of the most widely studied protocols [6]. The price of an individual's strategy $S_{i}$, with respect to the strategy profile $S$, is defined as $p_{i}(S)=\sum_{e \in S_{i}} \frac{p_{e}}{x_{e}(S)}$.

A profile $S$ is said to be a Nash equilibrium (NE) if no agent can improve their cost by a unilateral deviation from the profile, that is, for every player $i$ we have that for all $s_{i}-t_{i}$ paths $S_{i}^{\prime}$, it holds that $p_{i}(S) \leq p_{i}\left(S_{i}^{\prime}, S_{-i}\right)$. We consider two social cost functions: the sum-cost of a profile $S$, denoted by $s c_{\Delta}(S)=\sum_{i \in[n]} p_{i}(S)$, is the total cost to all agents in $S$, while the max-cost of a profile $S$, denoted by $m c_{\Delta}(S)=\max _{i \in[n]} p_{i}(S)$, is the maximum cost of any agent in $S$. We omit the subscript $\Delta$ if the game is clear from the context.

Note that a game $\Delta$ with undirected graph $G$ can be transformed into an equivalent game in directed graph $G^{\prime}$ using the following construction: Every undirected edge $\{u, v\}$ of $G$ is replaced by the directed edges $\left(u, x_{1}\right),\left(v, x_{1}\right)$, $\left(x_{1}, x_{2}\right),\left(x_{2}, u\right)$, and $\left(x_{2}, v\right)$, where $x_{1}$ and $x_{2}$ are two new nodes created for the transformation of $\{u, v\}$. The capacity and cost of $\left(x_{1}, x_{2}\right)$ are set equal to those of $\{u, v\}$, the remaining edges have infinite capacity and cost 0 . As a consequence of this transformation, any construction of undirected CCS games establishing a lower bound on the price of stability (or on the convergence time of BRD) automatically yields an equivalent construction of directed CCS games. Similarly, any upper bound on the price of stability proved for directed CCS games automatically yields the same upper bound for undirected CCS games. When it is clear from the context that we are considering undirected graphs, we also write undirected edges in the form $(u, v)$ instead of $\{u, v\}$.

Best Response Dynamics. If a strategy profile $S$ is not a NE, there will be a cheaper alternative to some player's path. We assume agents have full knowledge of the paths available to them, as well as their opponents' strategies, so they know the cost of all alternatives with respect to $S_{-i}$. Being self-motivated, players will update their strategies to the cheapest path available at any given point in what is known as best response dynamics (BRD). We do not specify the order in which updates are made, only that they are sequential and that the choice of strategy of the player making the update must be the best response to her opponents' current strategies. 
Existence of Nash Equilibria. The CCS games we consider fall into the class of congestion games studied by Monderer and Shapley [6], who show that all such games have pure Nash equilibria. They do this by defining a potential function $\Phi$, which in the context of our model is

$$
\Phi(S)=\sum_{e \in E} \sum_{i=1}^{x_{e}(S)} \frac{p_{e}}{i} .
$$

Note that $\Phi(S)$ is bounded by $\mathrm{H}(n)$ times the sum-cost of $S$. As players only make improving moves, best response dynamics will strictly reduce the potential of the solution with each step, meaning a profile cannot be revisited. As the strategy space of a game is finite, any sequence of updates will terminate at a profile where no player can make a unilateral improvement, which must be a NE.

Quality of Nash Equilibria. When measuring the quality of a NE we will compare its cost, by either the sum-cost or max-cost objective, to that of the optimal solution. The ratio between the objective value of the worst NE and the optimal objective value is called the price of anarchy, while the ratio between the objective value of the cheapest $\mathrm{NE}$ and the optimal objective value is called the price of stability, abbreviated to $\mathrm{PoA}$ and $\mathrm{PoS}$, respectively. We refer to the optimal objective value with respect to max-cost as $\mathrm{OPT}_{m c}$, and that with respect to sum-cost as $\mathrm{OPT}_{s c}$. Furthermore, we write $\operatorname{PoS}_{m c}(\Delta)$ for the price of stability with respect to max-cost, and similarly for the other cases. For a particular CCS game $\Delta$ whose set of Nash equilibria is denoted by $N E(\Delta)$, the prices of anarchy and stability with respect to max-cost are defined as

$$
\operatorname{PoA}_{m c}(\Delta)=\frac{\max _{S \in N E(\Delta)} m c_{\Delta}(S)}{O P T_{m c}(\Delta)} \operatorname{PoS}_{m c}(\Delta)=\frac{\min _{S \in N E(\Delta)} m c_{\Delta}(S)}{O P T_{m c}(\Delta)}
$$

with analogous calculations for sum-cost.

\section{Cost Increase and Convergence Time of BRD}

Best response dynamics are of interest both as a method to discover equilibria and for the effect they can have on an individual's cost. In potential games, the number of updates required to reach a stable solution is bounded by the cardinality of the strategy set, which is the set of all possible strategy combinations for all players. The size of the strategy set for a game depends on the size and topology of the underlying graph, the number of players, and the distribution of their source and sink nodes.

Examining the effect of BRD on a single player's cost, we now show that, with an arbitrary number of updates, an arbitrary increase in cost for that player is possible, within the limits of a factor $\mathrm{H}(n)$ increase in sum-cost. This result is of particular interest as it illustrates that within a game, a start profile which is cheap for a particular player is no guarantee of a good $N E$ for that individual. 
Theorem 1. There exists a symmetric CCS game $\Delta$ with 2 players and a start profile for $\Delta$ such that BRD increase the cost of a player to an arbitrary factor times the player's cost in the start profile.

Proof. For $m \in \mathbb{N}$ with $m \geq 3$, consider the CCS game $\Delta$ with $n=2$ players and underlying undirected graph $G=(V, E)$, defined as follows (see Figure 1 for an illustration for $m=3$ ):

$$
\begin{aligned}
& V=\left\{x_{i}, z_{i} \mid 0 \leq i \leq m\right\} \cup\left\{y_{i} \mid 1 \leq i \leq m\right\} \\
& E=\left\{\left(x_{i}, x_{i-1}\right),\left(z_{i}, z_{i-1}\right),\left(x_{i}, y_{i}\right),\left(y_{i}, z_{i-1}\right),\left(y_{i}, z_{i}\right) \mid 1 \leq i \leq m\right\}
\end{aligned}
$$

We denote the two players by $a$ and $b$. Their source and sink nodes are $s_{a}=z_{0}$, $t_{a}=x_{m}, s_{b}=x_{0}$, and $t_{b}=z_{m}$. We will discuss in the end how to make the game symmetric. A horizontal path from $x_{i}$ to $x_{j}$ for some $j \geq i$ is denoted by $x_{i} \rightarrow x_{j}$, and similarly for $z_{i} \rightarrow z_{j}$. (We will use this convention for denoting horizontal paths throughout the remainder of the paper.)

All edges have capacity 1 , except those connecting an $x$ node and a $y$ node, which have capacity 2 . Only edges incident with $y$ nodes have non-zero cost. For any node $y_{i}$, the costs of the connections to $z_{i-1}, z_{i}, x_{i}$ are denoted by $a_{i}, b_{i}$, $a b_{i}$, respectively. These costs are defined as follows (where $\varepsilon>0$ is a positive constant satisfying $\left.\varepsilon<1 / m^{2}\right)$ :

$$
\begin{array}{llll}
a_{1}=2^{m} & a_{i} & =2^{m}-2^{i}+2^{i-2}+1-i \varepsilon & \text { for } i>1 \\
b_{1}=1+\varepsilon & b_{i} & =0 \text { for } i>1 & \\
a b_{1}=0 & a b_{i} & =2^{i-1}+\varepsilon \text { for } i>1 &
\end{array}
$$

Let the start profile be $S=\left(\left(z_{0}, y_{1}, x_{1} \rightarrow x_{m}\right),\left(x_{0}, x_{1}, y_{1}, z_{1} \rightarrow z_{m}\right)\right)$. Our aim is to enable a sequence of $2 m-2$ best response moves such that the cost of player $b$ increases by an arbitrary factor (depending on $m$ ). In the start profile $S$, players $a$ and $b$ share the edge $\left(x_{1}, y_{1}\right)$ and their costs are $2^{m}$ and $1+\varepsilon$, respectively. Player $a$ 's best response to player $b$ 's strategy is now the path $z_{0}, z_{1}, y_{2}, x_{2} \rightarrow x_{m}$ with cost $a_{2}+a b_{2}=2^{m}-2-2 \varepsilon+2+\varepsilon=2^{m}-\varepsilon$, so player $a$ will update to that path. Player $b$ 's best response to $a$ 's new path is now the path $x_{0} \rightarrow x_{2}, y_{2}, z_{2} \rightarrow z_{m}$ with cost $a b_{2} / 2+b_{2}=1+\frac{\varepsilon}{2}$, so player $b$ will update to that path. As the edge $\left(x_{2}, y_{2}\right)$ is now shared, this reduces the cost of player $a$ to $2^{m}-1-2 \varepsilon+\varepsilon / 2$.

Claim. In the profile reached after $2(i-1)$ best response moves, for $2 \leq i \leq$ $m$, player $a$ uses path $z_{0} \rightarrow z_{i-1}, y_{i}, x_{i} \rightarrow x_{m}$ and player $b$ uses path $x_{0} \rightarrow$

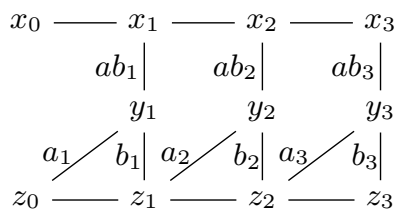

Fig. 1. Underlying graph of game in proof of Theorem 1 
$x_{i}, y_{i}, z_{i} \rightarrow z_{m}$. Player $a$ 's cost is $a_{i}+a b_{i} / 2=2^{m}-2^{i}+2^{i-2}+1-i \varepsilon+2^{i-2}+\varepsilon / 2=$ $2^{m}+2^{i-1}+1-2^{i}-i \varepsilon+\varepsilon / 2$, and player $b$ 's cost is $2^{i-2}+\varepsilon / 2$.

After $2(m-1)$ best response moves, player $a$ 's path is $\left(z_{0} \rightarrow z_{m-1}, y_{m}, x_{m}\right)$ with cost $2^{m-1}+1-m \varepsilon+\varepsilon / 2$ and player $b$ 's path is $\left(x_{0} \rightarrow x_{m}, y_{m}, z_{m}\right)$ with cost $2^{m-2}+\varepsilon / 2$. Denote this strategy profile by $S^{*}$. We claim that $S^{*}$ is a NE. First, note that player $a$ does not have an improving move: As the edges $\left(x_{m-1}, x_{m}\right)$ and $\left(z_{m}, y_{m}\right)$ have capacity 1 and are used by player $b$, player $a$ can reach $x_{m}$ only via the edges $\left(z_{m-1}, y_{m}\right)$ and $\left(y_{m}, x_{m}\right)$, and the path that $a$ uses in $S^{*}$ contains only zero-cost edges in addition to these two edges. Player $b$ 's only alternative paths that are potential improving moves are of the form $\left(x_{0} \rightarrow x_{i}, y_{i}, z_{i}, y_{i+1}, z_{i+1}, \ldots, y_{m-1}, z_{m-1}, z_{m}\right)$ for some $i<m-1$. Any such path would contain the edge $\left(z_{m-2}, y_{m-1}\right)$ with cost $a_{m-1}=2^{m}-2^{m-1}+$ $2^{m-3}+1-(m-1) \varepsilon=2^{m-1}+2^{m-3}+1-(m-1) \varepsilon>2^{m-2}+\varepsilon / 2$, so it would not be an improving move for $b$. Therefore, $S^{*}$ is a NE.

The cost of player $b$ is $1+\varepsilon$ in the start profile $S$ and $2^{m-2}+\varepsilon / 2$ in the NE $S^{*}$ that is reached by BRD from $S$. Hence, the cost of player $b$ has increased by a factor arbitrarily close to $2^{m-2}$. As $m$ can be chosen arbitrarily large, we have shown that the cost of a player can increase by an arbitrary factor during BRD.

Finally, we observe that the game can be made symmetric by adding edges $\left(s, z_{0}\right),\left(s, x_{0}\right),\left(x_{m}, t\right)$ and $\left(z_{m}, t\right)$ with cost 0 and capacity 1 , where $s$ and $t$ are two new nodes that represent the common source and destination, respectively. If player $a$ uses edges $\left(s, z_{0}\right)$ and $\left(x_{m}, t\right)$ and player $b$ uses edges $\left(s, x_{0}\right)$ and $\left(z_{m}, t\right)$ in the initial profile, this property must be maintained in every improving move, and BRD in this symmetric game behave in the same way as in the asymmetric game discussed above.

The symmetric CCS game defined in the proof of Theorem 1 has $n=2$ players and the convergence time of $\mathrm{BRD}$ is $2(m-1)$, where $m$ can be chosen arbitrarily large. This gives the following corollary, which is in contrast to the uncapacitated symmetric case where BRD converge in at most $n$ steps.

Corollary 1. There exists a symmetric CCS game and a strategy profile $S$ where $B R D$ converge to NE in an arbitrarily high number of steps, with respect to $n$.

\section{Price of Stability for Max-Cost}

In Section 5.1 we show that the $\operatorname{PoS}$ is $\Theta(n \log n)$ in the worst case for asymmetric CCS games. In Section 5.2 we show that the PoS is bounded by $n$ for symmetric CCS games.

\subsection{Asymmetric Games}

Theorem 2. There exists an asymmetric CCS game $\Delta$ with $n$ players and

$$
\operatorname{PoS}_{m c}(\Delta)=\Theta(n \log n)
$$


Using the potential function $\Phi$ defined in (1) in Section 3, it can be shown that the price of stability for max-cost is upper bounded by $O(n \log n)$ for any CCS game: Consider a strategy profile $S^{\prime}$ that minimises the max-cost. Let $C^{\prime}$ denote the sum-cost of $S^{\prime}$, and let $M^{\prime}$ denote the max-cost of $S^{\prime}$. It follows that the optimal max-cost $M^{\prime}$ satisfies $M^{\prime} \geq C^{\prime} / n$. Furthermore, the potential of $S^{\prime}$ is at most $\Phi\left(S^{\prime}\right) \leq C^{\prime} \cdot \mathrm{H}(n)=C^{\prime} / n \cdot n \mathrm{H}(n) \leq M^{\prime} n \mathrm{H}(n)$. BRD starting with $S^{\prime}$ converge to a NE $S^{*}$ without increasing the potential. Hence, the sum-cost of $S^{*}$, and therefore also the max-cost of $S^{*}$, is at most $M^{\prime} n \mathrm{H}(n)$ [4].

In the following, we will construct a game $\Delta$ with an odd number $n \geq 3$ of players where

$$
\min _{S^{*} \in N E(\Delta)} \max _{i \in[n]} p_{i}\left(S^{*}\right) \approx \frac{n}{2} \mathrm{H}(\lfloor n / 2\rfloor) \cdot O P T_{m c}(\Delta) .
$$

The construction uses parameters $m \in \mathbb{N}$ and $\varepsilon>0$, where $\varepsilon$ is sufficiently small, e.g., $\varepsilon<0.1$, and $m$ is sufficiently large. It is useful to think of $m$ as approaching infinity. Furthermore, for $2 \leq i \leq n$, let $k(i)$ denote the value $\lfloor(i+2) / 2\rfloor$. Let $\Delta$ be the CCS game with underlying graph $G=(V, E)$ defined as follows (see Figure 2 for an illustration of the structure of $G$ ):

$$
\begin{aligned}
& \left\{s_{1}, t_{1}, x_{[1, m]}, z_{[1, m]}\right\} \quad \cup \\
& V=\left\{s_{i}, t_{i}, z_{[i, 0]} \mid 1<i \leq n\right\} \quad \cup \\
& \left\{x_{[i, j]}, y_{[i, j]}, z_{[i, j]} \mid 1<i \leq n, 1 \leq j \leq m\right\} \\
& \left\{\left(s_{1}, x_{[1, m]}\right),\left(t_{1}, z_{[n, m]}\right),\left(x_{[1, m]}, z_{[1, m]}\right)\right\} \\
& E=\left\{\begin{array}{l}
\left(x_{[i, j]}, x_{[i, j-1]}\right),\left(x_{[i, j]}, y_{[i, j]}\right), \\
\left(y_{[i, j]}, z_{[i, j]}\right),\left(y_{[i, j]}, z_{[i, j-1]}\right),\left(z_{[i, j]}, z_{[i, j-1]}\right)
\end{array} \mid 1<i \leq n, 1<j \leq m\right\} \quad U \\
& \left\{\begin{array}{l}
\left(z_{[i, 0]}, z_{[i-1, m]}\right),\left(z_{[i, 0]}, z_{[i, 1]}\right),\left(z_{[i, 0]}, y_{[i, 1]}\right),\left(x_{[i, 1]}, y_{[i, 1]}\right), \\
\left(z_{[i, 1]}, y_{[i, 1]}\right),\left(x_{[1, m]}, x_{[i, 1]}\right),\left(z_{[i, 0]}, s_{i}\right),\left(s_{i}, z_{[1, m]}\right),\left(t_{i}, x_{[i, m]}\right)
\end{array} \mid 1<i \leq n\right\} \\
& c(e)=\left\{\begin{array}{l}
n \text { if } e=\left(x_{[1, m]}, z_{[1, m]}\right) \\
2 \text { if } e=\left(x_{[i, j]}, y_{[i, j]}\right): 1<i \leq n, 1 \leq j \leq m \\
1 \text { otherwise }
\end{array}\right. \\
& p(e)= \begin{cases}2+2 \varepsilon & \text { if } e=\left(x_{[1, m]}, z_{[1, m]}\right) \\
\frac{1}{k(i) 2^{j-1}} & \text { if } e=\left(x_{[i, j]}, y_{[i, j]}\right): 1<i \leq n, 1 \leq j \leq m \\
\mathrm{H}(k(i))-\frac{3}{k(i) 2^{j}}+\frac{\varepsilon}{k(i) m+j} & \text { if } e=\left(y_{[i, j]}, z_{[i, j]}\right): 1<i \leq n, 1 \leq j \leq m \\
\frac{\varepsilon}{k(i)+j} & \text { if } e=\left(y_{[i, j]}, z_{[i, j-1]}\right): 1<i \leq n, 1 \leq j \leq m \\
0 & \text { otherwise }\end{cases}
\end{aligned}
$$

The source and sink of player $i$, for $1 \leq i \leq n$, are $s_{i}$ and $t_{i}$, respectively. We refer to the nodes of the form $x_{[i, j]}$ as the $x$-row, to the nodes of the form $y_{[i, j]}$ as the $y$-row, and to the nodes of the form $z_{[i, j]}$ as the $z$-row. We divide the main part of the graph into grids as follows: For any $i \geq 2$, the $i$-th grid 


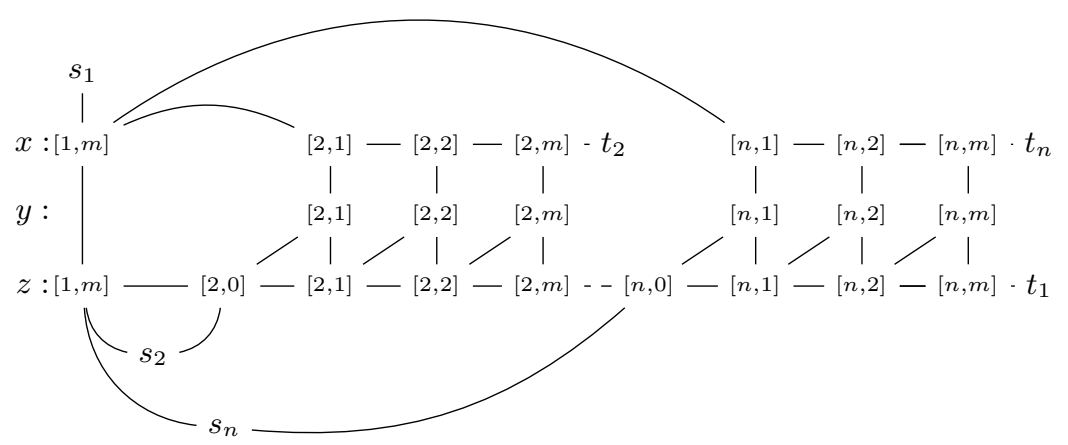

Fig. 2. Game $\Delta$ with $\operatorname{PoS}_{m c}(\Delta)=\Theta(n \log n)$ for $m=3$

is the induced subgraph of all $x, y, z$ nodes with subscript $[i, j]$ for any $j$. Note that the edge costs in pairs of consecutive grids, namely the $(2 k-2)$-th and $(2 k-1)$-th grid, are the same for $2 \leq k \leq\lceil n / 2\rceil$. For fixed $i$ and $j$, we refer to the subgraph induced by $x_{[i, j]}, y_{[i, j]}, z_{[i, j]}$ and $z_{[i, j-1]}$ as column $j$ of the $i$-th grid. For simplicity we will refer to the costs of the connections from $y_{[i, j]}$ to $x_{[i, j]}, z_{[i, j]}, z_{[i, j-1]}$ as $a b_{[k(i), j]}, a_{[k(i), j]}, b_{[k(i), j]}$, respectively.

Lemma 1. $O P T_{m c}(\Delta) \leq \frac{2+2 \varepsilon}{n}$

Proof. Consider the profile $S^{\prime}$ where player 1 uses the path $\left(s_{1}, x_{[1, m]}, z_{[1, m]} \rightarrow\right.$ $\left.z_{[n, m]}, t_{1}\right)$ and player $i$, for $2 \leq i \leq n$, uses the path $\left(s_{i}, z_{[1, m]}, x_{[1, m]}, x_{[i, 1]} \rightarrow\right.$ $\left.x_{[i, m]}, t_{i}\right)$. All $n$ players share the edge $\left(x_{[1, m]}, z_{[1, m]}\right)$ and use no other edge with non-zero cost. Each player has cost $(2+2 \varepsilon) / n$. The optimal max-cost is therefore at most $(2+2 \varepsilon) / n$.

Lemma 2. $\min _{S \in N E(\Delta)} m c_{\Delta}(S) \geq a_{[k(n), m]} \geq \mathrm{H}(\lfloor n / 2\rfloor)-\frac{3}{k(n) 2^{m}}+\frac{\varepsilon}{(k(n)+1) m}$

Proof (Sketch).

We claim that player 1 must pass through $x_{[n, m]}$ or $x_{[n-1, m]}$ and hence use edges $\left(x_{[i, m]}, y_{[i, m]}\right)$ and $\left(y_{[i, m]}, z_{[i, m]}\right)$ for $i=n-1$ or for $i=n$ in any NE (note that using edges $\left(y_{[i, m]}, z_{[i, m-1]}\right)$ and $\left(z_{[i, m-1]}, z_{[i, m]}\right)$ would block player $i$ from reaching $\left.t_{i}\right)$, thus paying at least $a_{[k(n-1), m]}=a_{[k(n), m]}$. To establish that player 1 must pass through $x_{[n, m]}$ or $x_{[n-1, m]}$ in any NE, we show that in all other cases some player has an improving move.

Let $S$ be a NE. Assume that the path $S_{1}$ of player 1 does not pass through $x_{[n, m]}$ or $x_{[n-1, m]}$. Consider the last $x$ node (i.e., node in the $x$-row) that the path $S_{1}$ of player 1 visits. Let $x_{[i, j]}$ be that node. Note that $i<n-1$ or $j<m$. If $i \geq 2$, note that $x_{[i, j]}$ must be followed directly by $y_{[i, j]}$ and $z_{[i, j]}$ on $S_{1}$ because using the subpath $\left(y_{[i, j]}, z_{[i, j-1]}, z_{[i, j]}\right)$ would block player $i$ from reaching her destination $t_{i}$. If $i=1$, the path $S_{1}$ must use the edge $\left(x_{[1, m]}, z_{[1, m]}\right)$. There are three possible cases for the location of the last $x$ node on $S_{1}$, and it can be shown that some player has an improving move in each case. 
Case 1: The last $x$ node on $S_{1}$ is $x_{[1, m]} . S_{1}$ must travel from $x_{[1, m]}$ to $z_{[1, m]}$ and then reach $t_{1}$ by visiting all nodes $z_{[i, j]}$ from left to right, possibly visiting some $y$ nodes in between adjacent $z$ nodes. One can show that no other player $i$ can share the edge $e_{1}=\left(x_{[1, m]}, z_{[1, m]}\right)$ with player 1 , as player $i$ would have an improving move via $z_{[i, 0]}$ in that case due to $\frac{2+2 \varepsilon}{i}>a b_{[k(i), 1]}+b_{[k(i), 1]}$. Hence, player 1 pays the full price $2+2 \epsilon$ for $e_{1}$ and one can show that she has an improving move via $x_{[2,1]}$.

Case 2: The last $x$ node on $S_{1}$ is $x_{[i, j]}$ for some $i \geq 2, j<m$. One can use $b_{[k(i), j]}+a b_{[k(i), j]} / 2>b_{[k(i), j+1]}+a b_{[k(i), j+1]}$ to show that player $i$ passes through $\left(x_{[i, j+1]}, y_{[i, j+1]}\right)$ (except in one case that can be handled differently) and $a_{[k(i), j]}+a b_{[k(i), j]}>a_{[k(i), j+1]}+a b_{[k(i), j+1]} / 2$ to show that player 1 then has an improving move to column $j+1$ of the $i$-th grid.

Case 3: The last $x$ node on $S_{1}$ is $x_{[i, m]}$ for $2 \leq i<n-1$. Player 1 must continue from $x_{[i, m]}$ via $\left(x_{[i, m]}, y_{[i, m]}, z_{[i, m]}, z_{[i+1,0]}\right)$ and pass through the $i^{\prime}$-th grid for all $i \leq i^{\prime} \leq n$ to reach $t_{1}$. Edges in any such grid can therefore only be used by player 1 and player $i^{\prime}$. Let $i^{\prime} \in\{i+1, i+2\}$ be such that player $i^{\prime}$ reaches the $i^{\prime}$-th grid via the edge $\left(s_{i^{\prime}}, z_{\left[i^{\prime}, 0\right]}\right)$. One can show that such an $i^{\prime}$ exists. Player 1 must visit $z_{\left[i^{\prime}, 0\right]}$. Using $a_{[k(i), m]}+a b_{[k(i), m]} / 2>a_{\left[k\left(i^{\prime}\right), 1\right]}+a b_{\left[k\left(i^{\prime}\right), 1\right]} / 2$ for $i^{\prime} \in\{i+1, i+2\}$, player 1 has an improving move by choosing a path starting with $\left(s_{1}, x_{[1, m]}, x_{\left[i^{\prime}, 1\right]}, y_{\left[i^{\prime}, 1\right]}\right)$.

Proof (of Theorem 2). We have constructed a CCS game $\Delta$ with an optimal strategy profile with a max-cost of at most $\frac{2+2 \varepsilon}{n}$ by Lemma 1 , while the max-cost of the best $N E$ is arbitrarily close to $\mathrm{H}(\lfloor n / 2\rfloor)$ by Lemma 2 . Hence, $P o S_{m c}(\Delta)$ approaches $n \mathrm{H}(\lfloor n / 2\rfloor) /(2+\varepsilon)=\Theta(n \log n)$ arbitrarily closely.

\subsection{Symmetric Games}

We now consider the case where all players have the same source and sink, and find that the upper bound of $O(n \log n)$ on the price of stability is not tight. First, we note that BRD starting with a strategy profile with optimal max-cost can increase the max-cost by a factor of $\Omega(n \log n)$ even in the symmetric case. The game constructed for the proof of Theorem 2 in Section 5.1 can be made symmetric by attaching a common source $s$ that is made adjacent to $\left\{s_{1}, \ldots, s_{n}\right\}$ and a common destination $t$ that is made adjacent to $\left\{t_{1}, \ldots, t_{n}\right\}$. Starting with the strategy profile where player $i$ visits $s_{i}$, the edge $\left(x_{[1, m]}, z_{[1, m]}\right)$, and $t_{i}$, BRD will converge to a NE that corresponds to a $\mathrm{NE}$ for the asymmetric game and has max-cost $\Omega(n \log n)$ times the optimal max-cost. Hence, in order to bound the price of stability in the symmetric case, we cannot use the standard proof technique of analysing BRD starting with the optimal strategy profile. Instead, we use a different approach that may be of independent interest. We iteratively discard a single expensive path from the NE reached by BRD and recombine the remaining $n-1$ paths with the optimal strategy profile, until a NE with small max-cost is obtained. In this way we are able to show that for every symmetric CCS game $\Delta$ there is always a NE where no player pays more than $n$ times $O P T_{m c}(\Delta)$. 
Theorem 3. For any symmetric CCS game $\Delta$ in directed or undirected networks, $\operatorname{PoS}_{m c}(\Delta) \leq n$.

Proof. We present the proof for directed networks. The result for undirected networks follows using the standard transformation of an undirected network into an equivalent directed network discussed in Section 3.

Let $\Delta$ be a symmetric CCS game with directed graph $G=(V, E), n$ players, common source $s$ and common destination $t$. Let $S$ be the optimal strategy profile with respect to max-cost. Without loss of generality, we can scale the edge costs so that the sum-cost of $S$ is $n$. This implies $m c(S) \geq 1$.

Consider the NE $S^{*}$ that is obtained from $S$ using BRD. If $m c\left(S^{*}\right) \leq n$, then $\operatorname{PoS}(\Delta) \leq n$ and we are done. Otherwise, we have $n<m c\left(S^{*}\right) \leq s c\left(S^{*}\right) \leq$ $\Phi\left(S^{*}\right)<\Phi(S)$. Let $\Phi(S)=n+\alpha$ and $m c\left(S^{*}\right)=n+\beta$ for some $\alpha, \beta>0$, and let $\Phi\left(S^{*}\right)=\Phi(S)-\delta$ for some $\delta>0$. Note that $0<\beta \leq \alpha-\delta$. The following table illustrates these quantities:

\begin{tabular}{l|r|c} 
& $m c$ & $\Phi$ \\
\hline$S$ & $\geq 1$ & $n+\alpha$ \\
$S^{*}$ & $n+\beta$ & $n+\alpha-\delta$
\end{tabular}

Now consider the $(n-1)$-player profile $S_{-1}^{*}$ that consists of the $n-1$ cheapest (in terms of cost to the respective player) strategies in $S^{*}$. As the change in potential function equals the cost to an individual player when making some change in strategy, we have that $\Phi\left(S_{-1}^{*}\right)=\Phi\left(S^{*}\right)-m c\left(S^{*}\right)=n+\alpha-\delta-(n+\beta)=\alpha-\beta-\delta$.

We construct a new $n$-player strategy profile $S^{\prime}$ by combining $S$ and $S_{-1}^{*}$ using an augmentation step in a suitably defined flow network. (We refer the reader to [1] for background on network flow, residual networks, and augmenting paths.) First, define the capacitated network $\bar{G}=(V, \bar{E})$ from $G=(V, E)$ by letting $\bar{E}=\left\{e \in E \mid x_{e}(S)>0\right.$ or $\left.x_{e}\left(S_{-1}^{*}\right)>0\right\}$ and setting the capacity $\bar{c}(e)$ for each $e \in \bar{E}$ to $\bar{c}(e)=\max \left\{x_{e}(S), x_{e}\left(S_{-1}^{*}\right)\right\}$. The strategy profile $S_{-1}^{*}$ induces a flow $f$ of value $n-1$ from $s$ to $t$ in $\bar{G}$. The network $\bar{G}$ admits a flow of value $n$ from $s$ to $t$ as the profile $S$ induces such a flow. Hence, the residual network $\bar{G}_{f}$ of $\bar{G}$ with respect to flow $f$ admits an augmenting path $P$ from $s$ to $t$. Let $f^{\prime}$ be the flow of value $n$ obtained by augmenting $f$ with $P$. Decompose the flow $f^{\prime}$ into $n$ paths from $s$ to $t$, and let $S^{\prime}$ be the strategy profile corresponding to these $n$ paths.

In going from $f$ to $f^{\prime}$, the flow on any edge increases by at most 1 , and every edge on which the flow increases satisfies $x_{e}(S)>0$. Let $X$ be the set of edges on which the flow increases. Observe that $\Phi\left(S^{\prime}\right) \leq \Phi\left(S_{-1}^{*}\right)+p(X)$, because increasing the number of players on an edge $e$ by 1 adds at most $p(e)$ to the potential. As $X \subseteq\left\{e \in E \mid x_{e}(S)>0\right\}$, we have $p(X) \leq s c(S)=n$ and hence $\Phi\left(S^{\prime}\right) \leq \alpha-\beta-\delta+n<\Phi\left(S^{*}\right)$.

Let $S^{* *}$ be the NE obtained from $S^{\prime}$ via BRD. Note that $\Phi\left(S^{* *}\right) \leq \Phi\left(S^{\prime}\right)<$ $\Phi\left(S^{*}\right)$. If $m c\left(S^{* *}\right) \leq n$, we have found a NE with max-cost at most $n$ times the optimal max-cost and we are done. Otherwise, we can repeat the construction that we used to create $S^{* *}$ from $S^{*}$, but starting with $S^{* *}$ in place of $S^{*}$. Each time we repeat the construction and obtain a $\mathrm{NE}$ with max-cost greater than $n$, 
that NE has strictly smaller potential than the previous NE. As the number of strategy profiles is finite, we must eventually obtain a NE whose max-cost is at most $n$. This shows $\operatorname{PoS}_{m c}(\Delta) \leq n$.

\section{Further Research}

As we have shown in Corollary 1 that the convergence time of BRD cannot be bounded by any function of $n$ for symmetric CCS games, an interesting question is in what other settings it is possible for BRD convergence to be unbounded in $n$. In our setting the effect of capacitated edges is to allow the reachable strategy space of an individual player to be limited by their opponent's choice of path, thus increasing the number of states which can be passed before a stable solution is reached. It may be interesting to identify other games where this behaviour could be observed.

To prove that the price of stability with respect to max-cost is bounded by $n$ for symmetric CCS games, we iteratively combined a NE with large max-cost with the optimal strategy profile. It would be interesting to explore whether this method could be turned into an efficient procedure for constructively finding a good NE. As the approach mainly relies on arguments about the reduction in potential of the strategy profiles constructed, it may be possible to apply it to other potential games.

\section{References}

1. Ahuja, R.K., Magnanti, T.L., Orlin, J.B.: Network Flows: Theory, Algorithms, and Applications. Prentice Hall, New Jersey (1993)

2. Anshelevich, E., Dasgupta, A., Kleinberg, J.M., Tardos, É., Wexler, T., Roughgarden, T.: The price of stability for network design with fair cost allocation. SIAM J. Comput. 38(4), 1602-1623 (2008)

3. Disser, Y., Feldmann, A.E., Klimm, M., Mihalák, M.: Improving the $H_{k}$-bound on the price of stability in undirected shapley network design games. Theor. Comput. Sci. 562, 557-564 (2015)

4. Feldman, M., Ron, T.: Capacitated network design games. In: 5th International Symposium on Algorithmic Game Theory (SAGT 2012). pp. 132-143. LNCS 7615, Springer (2012)

5. Koutsoupias, E., Papadimitriou, C.H.: Worst-case equilibria. In: Proceedings of the 16th Annual Symposium on Theoretical Aspects of Computer Science (STACS 99). pp. 404-413. LNCS 1563, Springer (1999)

6. Monderer, D., Shapley, L.S.: Potential games. Games and Economic Behavior 14, 124-143 (1996)

7. Nisan, N., Roughgarden, T., Tardos, E., Vazirani, V.V.: Algorithmic Game Theory. Cambridge University Press, New York, NY, USA (2007)

8. Roughgarden, T., Tardos, É.: How bad is selfish routing? Journal of the ACM 49(2), 236-259 (2002)

9. Schulz, A.S., Moses, N.E.S.: On the performance of user equilibria in traffic networks. In: Proceedings of the Fourteenth Annual ACM-SIAM Symposium on Discrete Algorithms (SODA '03). pp. 86-87 (2003) 